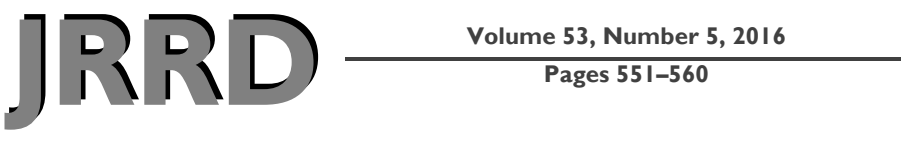

\title{
Balance confidence and activity of community-dwelling patients with transtibial amputation
}

\author{
Alena Mandel, MScOT; ${ }^{1}$ Kailan Paul, MScOT; ${ }^{1}$ Ruby Paner, BSc ${ }^{2}$ Michael Devlin, MD, FRCPC ${ }^{3}$ Steven \\ Dilkas, MD, FRCPC; ${ }^{3-4}$ Tim Pauley, MSc $^{2 *}$ \\ ${ }^{1}$ Department of Occupational Science and Occupational Therapy, University of Toronto, Toronto, ON, Canada; \\ ${ }^{2}$ Nathan Clinical Research Institute, West Park Healthcare Centre, Toronto, ON, Canada; ${ }^{3}$ Amputee Rehabilitation \\ Services, West Park Healthcare Centre, Toronto, ON, Canada; ${ }^{4}$ Division of Physical Medicine and Rehabilitation, \\ Department of Medicine, University of Toronto, ON, Canada
}

\begin{abstract}
The purpose of this study was to examine the relationship between balance confidence and community-based physical activity. Twenty-two community-dwelling patients with right or left unilateral transtibial amputation who reported no falls in the past 6 mo completed the Activities-Specific Balance Confidence Scale $(\mathrm{ABC})$ and wore a StepWatch Activity Monitor for 7 consecutive $d$ in the community. Subjects were subsequently stratified as low $(<3,000 \mathrm{steps} / \mathrm{d})$ or high $(>/=3,000 \mathrm{steps} /$ d) activity groups. Balance confidence was significantly lower among the low activity weekday group (LAG) $(70.8+/-$ 12.0 vs $88.9+/-8.7, t(20)=3.97, p=0.001)$. Further, correlation analysis revealed a positive correlation between $\mathrm{ABC}$ score and step total $(r=0.55, p<0.01)$. It is unknown whether the LAG limited ambulation as an intentional strategy of fall-risk avoidance. Although clinicians routinely inquire about falls in the community among patients with lower-limb amputation, the results of this study emphasize the importance of contextualizing recent fall history relative to activity level. Clinicians can use this contextual information when considering the inclusion of appropriate fall-risk mediation strategies relative to activity levels and counseling patients on the benefits of physical exercise for maintaining functional capacity and general health.
\end{abstract}

Key words: ambulation, amputation, amputees, artificial limbs, balance confidence, fall risk, lower limb, mobility capacity, physical activity, prosthesis.

\section{INTRODUCTION}

Individuals with a lower-limb (LL) amputation face a number of challenges reintegrating into the community following inpatient rehabilitation. One of these challenges is the ability to sustain a healthy level of physical activity. Previous research has demonstrated that individuals with LL amputation engage in less physical activity compared to those without any known LL impairments [1]. A second challenge faced by individuals with LL amputation is maintaining engagement in various leisure and productive activities. Previous research using subjective assessments has found that this population participates in fewer leisure activities after amputation compared to before amputation, and this decreased participation persists following inpatient rehabilitation [2-3].

Abbreviations: $\mathrm{ABC}=$ Activities-Specific Balance Confidence Scale, $\mathrm{ANOVA}=$ analysis of variance, $\mathrm{HAG}=$ high activity group, $\mathrm{LAG}=$ low activity group, $\mathrm{LL}=$ lower-limb, SAM = StepWatch Activity Monitor, SPM = steps per minute, TFA = transfemoral amputation, TTA $=$ transtibial amputation.

*Address all correspondence to Tim Pauley, MSc; West Park Healthcare Centre, 82 Buttonwood Ave, Toronto, ON, Canada M6M 2J5; 416-243-3600 ext. 2628; fax: 416-2433747. Email: Tim.paulev@westpark.org

http://dx.doi.org/10.1682/JRRD.2015.03.0044 
Individuals with LL amputation also are at a high risk for falls. Previous research has found that 58 percent of individuals with a unilateral amputation and 27 percent of individuals with a bilateral amputation have experienced a fall within the past 12 mo [4]. Within the small pool of literature studying falls experienced by individuals with LL amputation, research has explored intrinsic factors that may contribute to an increased fall risk, including gait differences and balance abnormalities [5], as well as fear of falling and balance confidence [6].

To our knowledge, only one study has examined both the aforementioned challenges of activity level (divided into mobility capacity/performance and participation in social activity) and fall history with both faller and nonfaller individuals with LL amputation [6]. In this context, nonfallers included those individuals with no history of previous falls. The results from this study found that balance confidence is positively related to mobility capacity/ performance and participation in social activities, regardless of fall status. The results of a follow-up study further highlighted the importance of balance confidence. The study determined that decreased balance confidence is a psychological factor related to decreased mobility and participation in community activities [7]. These studies have contributed to a greater understanding of the relationship between balance confidence and activity level between people with and without a fall history. Thus, we have an opportunity to examine nonfallers as a principal study group for the purpose of objectively exploring activity patterns and balance confidence.

A lack of focus on activity levels of nonfallers exclusively is common. The few studies investigating fall history have primarily explored the physical and psychological factors that may contribute to falls, as opposed to the characteristics of nonfallers [4-8]. Nonfallers may be understudied relative to those with a history of previous falls because researchers and professionals may have assumed that nonfallers experience fewer challenges with ambulation and/or activity within the community and thus are at low risk of falling. As a result, there are currently no studies exclusively exploring the activity level and balance confidence of the nonfaller LL amputation population.

Given the lack of exclusive examination of nonfallers and the practical challenges associated with prolonged objective measurement of fall occurrence in the community, one cannot conclude definitively that self-reported nonfaller individuals with LL amputation have not experienced a fall as a result of effective use of their prosthetic device for community ambulation. Conversely, it is equally possible that a subgroup of nonfallers exist whose nonfaller status is due as much to an intentional strategy of nonengagement in physical activity (thus actively lessening their exposure to fall risk as a result of fear of falling) [9] as it is to sufficient ambulatory capacity. Indeed, it has been demonstrated that among community-dwelling patients with LL amputation, those with higher balance ability were at an increased risk of experiencing a fall, while those with the poorest balance ability actively avoid fall risk by using a wheelchair or limiting activities [10]. The purpose of the current study, therefore, was to explore the relationship between community-based physical activity and balance confidence among a group of nonfaller patients with LL amputation; thus, we set out to test the null hypothesis that there is no relationship between self-perceived balance confidence and community-based physical activity.

\section{METHODS}

\section{Subjects}

Subjects were included in the study if they had undergone unilateral transtibial amputation (TTA), had been using a prosthesis for at least 6 mo, were independently ambulatory, were over $40 \mathrm{yr}$ of age, and were able to complete all baseline testing procedures. Additionally, subjects must also have met the criteria of nonfaller in the past 6 mo [11]. A fall was defined as landing on the floor or the ground unintentionally [9,12]. If individuals disclosed that they had fallen within the previous 6 mo, they were excluded from the study and appropriate services were coordinated as required.

\section{Procedures}

This study utilized a longitudinal design. Data were captured in 2014. Winter months were avoided to control for potential confounding factors associated with weather effects. That is, one could reasonably assume that community activity would drop in the winter as a result of weather patterns alone. All study subjects were current patients of West Park Healthcare Centre receiving outpatient rehabilitation. Subjects were recruited either through the mail or during their outpatient visit. Those subjects recruited via mail were prescreened for inclusion in the study prior to mail contact and subsequently contacted via telephone to confirm that they were interested in participating in the study and that they met all inclusion 
criteria. At this time, interested subjects also completed a demographics questionnaire and the Activities-Specific Balance Confidence Scale (ABC) [13]. Subjects were sent a StepWatch Activity Monitor (SAM, Orthocare Innovations; Mount Lake, Washington) via regular postal service with text and pictorial instructions regarding the proper method for attaching the device to the prosthetic leg as well as a self-addressed stamped envelope in which to return the SAM after wearing it for 7 consecutive d. In spite of these measures, two subjects attached the SAM upside down, which yielded no useful data. In response, researchers sent out the SAMs to these subjects a second time. Researchers also followed up with subsequent subjects roughly $1 \mathrm{~d}$ after they were expected to have received the SAM to ensure that the subjects had properly attached the device. Subjects were reminded to engage in the same amount of physical activity that was typical for them in a normal day to avoid risk of physical injury and to ensure that the SAM was capturing their usual amount of daily activity.

Those subjects recruited during their outpatient visit were referred to the research assistant by the clinic receptionist. The research assistant reviewed the study information sheet, obtained written consent, and attached the SAM to the prosthetic leg. At this time, these subjects also completed a demographics questionnaire and the ABC. In addition, subjects were provided with text and pictorial instructions regarding the proper method for reattaching the SAM to the prosthetic leg as well as a self-addressed stamped envelope in which to return the SAM after wearing it for 7 consecutive $d$.

\section{Apparatus}

\section{Physical Activity Monitor}

Community-based physical activity was captured with a SAM that was attached to the prosthetic leg of study subjects for 7 consecutive d. The SAM was chosen as an objective measure of activity because previous research has demonstrated the inability of patients with LL amputation to accurately self-report their activity levels [14-16]. Further, the SAM has been shown to have good test-retest reliability among both older adult [17] and stroke patients [18] when measured over a $3 \mathrm{~d}$ monitoring period; it also correlates well with the 2 min walk test among patients with LL amputations [19]. The SAM measures ambulatory activity by recording the number of steps taken per day. In addition, the SAM allows for eval- uation of the quality of stepping activity by quantifying the rate of steps taken per minute in designated stepfrequency categories. The SAM was programmed based on subjects' height and set to record for $15 \mathrm{~d}$ to ensure that the device captured a full $7 \mathrm{~d}$ of ambulatory activity while accounting for dispatch and return postal service time.

\section{Balance Confidence}

The $\mathrm{ABC}$ was used to measure an individual's selfperceived balance confidence. The $\mathrm{ABC}$ is a 16-item self-report questionnaire that requires subjects to imagine themselves performing daily activities and rate their perceived level of balance confidence on a scale from 0 percent (least confident) to 100 percent (most confident) [20]. This measure has been shown to be reliable when used with individuals with LL amputation [21-22] and has been administered over the telephone [13].

\section{Analysis}

For purposes of the current study, subjects were sorted into high (HAG, $\geq 3,000$ steps/d) and low activity groups (LAG, $<3,000$ steps/d). An apparently appropriate delineator of 3,000 steps/d was selected a priori based on the average step count for individuals with a TTA (3,000 steps/d with their prosthetic device) reported by previous research [15]. The 3,000 steps/d threshold quantifies the stepping activity of both legs combined (step total). Because the SAM captures data from only one leg, this data translates to a measured stepping activity equivalent of 1,500 steps. The singleleg data was utilized for purposes of analysis; however, data reported herein are for both legs combined as is consistent with previously published data [15]. To describe the quality of stepping, data were analyzed not only as the mean daily total number of steps but also as a feature of the rate of stepping per minute. Thus, similar to a previously published study of patients with LL amputation [19], step total was further categorized within low ( $<16$ steps per min $[\mathrm{SPM}]$ ), medium (16-40 SPM), and high ( $>40$ SPM) step-frequency categories and analyzed in absolute and relative terms. Because weekday activities may be more routine (e.g., work, school, volunteering) than weekend activities, activity data for weekdays and weekends were segregated for separate analyses. Weekday activity was the primary focus of the analyses for this study. For those subjects who received the SAM on Tuesday, Wednesday, Thursday, or Friday, nonconsecutive weekday data from $5 \mathrm{~d}$ were combined. The $t$-test and chi-square statistics were used to compare continuous 
and categorical data, respectively, for HAG and LAG segmented by weekday and weekend activity data. Correlation analysis was used to assess the relationship between balance confidence and stepping activity. SPSS software (Windows, version 22, IBM Corp; Armonk, New York), was used to conduct the analyses.

\section{RESULTS}

Subjects' demographics and clinical characteristics are included in Table 1. We recruited 14 male and 8 female community-dwelling patients with right or left unilateral TTA (age 61.4 \pm 7.6 yr). Time since amputation was $3.2 \pm 3.2 \mathrm{yr}$. The majority of patients had undergone amputation secondary to diabetes or peripheral vascular disease. Eighteen subjects used some manner of walking aid, and four were able to ambulate with no

Table 1.

Demographic and clinical data of study participants $(N=22)$. Data presented as $n(\%)$ or mean \pm standard deviation.

\begin{tabular}{lc}
\hline \multicolumn{1}{c}{ Characteristic } & Subject Data \\
\hline Sex & $14(63.6)$ \\
Male & $8(36.4)$ \\
Female & $61.4 \pm 7.6$ \\
Age (yr) & $3.2 \pm 3.2$ \\
Time Since Amputation (yr) & \\
Cause of Amputation & $14(63.6)$ \\
Diabetes/Peripheral Vascular Disease & $3(13.6)$ \\
Motor Vehicle Accident & $3(13.6)$ \\
Accident, Other & $2(9.1)$ \\
Cancer & \\
Walking Aid & $13(59.1)$ \\
Cane & $4(18.2)$ \\
None & $4(18.2)$ \\
Rollator & $1(4.5)$ \\
Crutches & \\
Living Setting & $8(36.4)$ \\
Apartment & $6(27.3)$ \\
House, One Story & $6(27.3)$ \\
House, Two Story & $2(9.1)$ \\
Seniors' Apartment Complex & \\
Living Arrangement & $12(54.5)$ \\
With Spouse & $4(18.2)$ \\
With Family (spouse and children) & $3(13.6)$ \\
Alone & $3(13.6)$ \\
With Parent(s) & \\
\hline \hline
\end{tabular}

assistive device. Twenty subjects lived in an apartment or house; 19 lived with family. There were no differences between groups in terms of age, total step count, or ABC scores for those subjects recruited via mail or during their outpatient visit ( $p>0.05$ for both).

Stratification of the entire sample into HAG and LAG resulted in a slight shift of group assignment when segmenting weekday and weekend data. Stratification of subjects on weekday activity data identified $10 \mathrm{HAG}$ and 12 LAG subjects. However, two LAG subjects were recategorized as HAG subjects based on weekend data because they increased their mean daily steps from below to above the 3,000-step threshold, and two HAG subjects were recategorized as LAG subjects based on weekend data. One subject categorized as HAG based on weekday data did not wear the SAM on the weekend.

There were no significant differences between groups in terms of age or time since amputation for either the weekday or weekend data $(p>0.05$ for all), though there was a trend toward a lower mean age for the HAG compared to the LAG for weekdays $(58.2 \pm 7.7$ versus $64.0 \pm 6.8, t(20)=1.88, p=0.08)$. Further, there were no significant differences between groups in terms of cause of amputation, use of a walking aid, or living arrangements $(p>0.05$ for all).

SAM and ABC data are presented in Table 2 for the HAG and LAG segmented by weekday and weekend activity. The ABC data indicated a greater balance confidence among the HAG compared to the LAG, regardless of whether the sample was segmented by weekdays $(88.9 \pm$ 8.7 versus $70.8 \pm 12.0, t(20)=3.97, p=0.001)$ or weekend days $(85.6 \pm 10.1$ versus $73.0 \pm 14.2, t(19)=2.25, p<0.05)$. Given the unanticipated trend toward a lower mean age for the HAG compared to the LAG for weekday activity, we conducted a univariate analysis of variance (ANOVA) with age entered as a covariate. The results of the ANOVA confirmed the statistically significant difference between groups in $\mathrm{ABC}$ score $(F(1,19)=16.84, p=0.001)$.

Correlation analysis revealed a positive correlation between $\mathrm{ABC}$ score and step total $(r=0.55, p<0.01)$ for weekday data. Individual subject level data for $\mathrm{ABC}$ score and step total is depicted graphically in the Figure. Further, we found a positive correlation between $\mathrm{ABC}$ score and the proportion of stepping in the high step-frequency category ( $>40$ SPM, $r=0.58, p<0.01$ ). Conversely, we found a negative correlation between $\mathrm{ABC}$ score and the proportion of stepping in the low step-frequency category ( $<16$ SPM $, r=-0.56, p<0.01)$. We observed a similar 
Table 2.

Activities-Specific Balance Confidence Scale (ABC) and StepWatch Activity Monitor data for the high and low activity groups segmented by weekday and weekend activity.

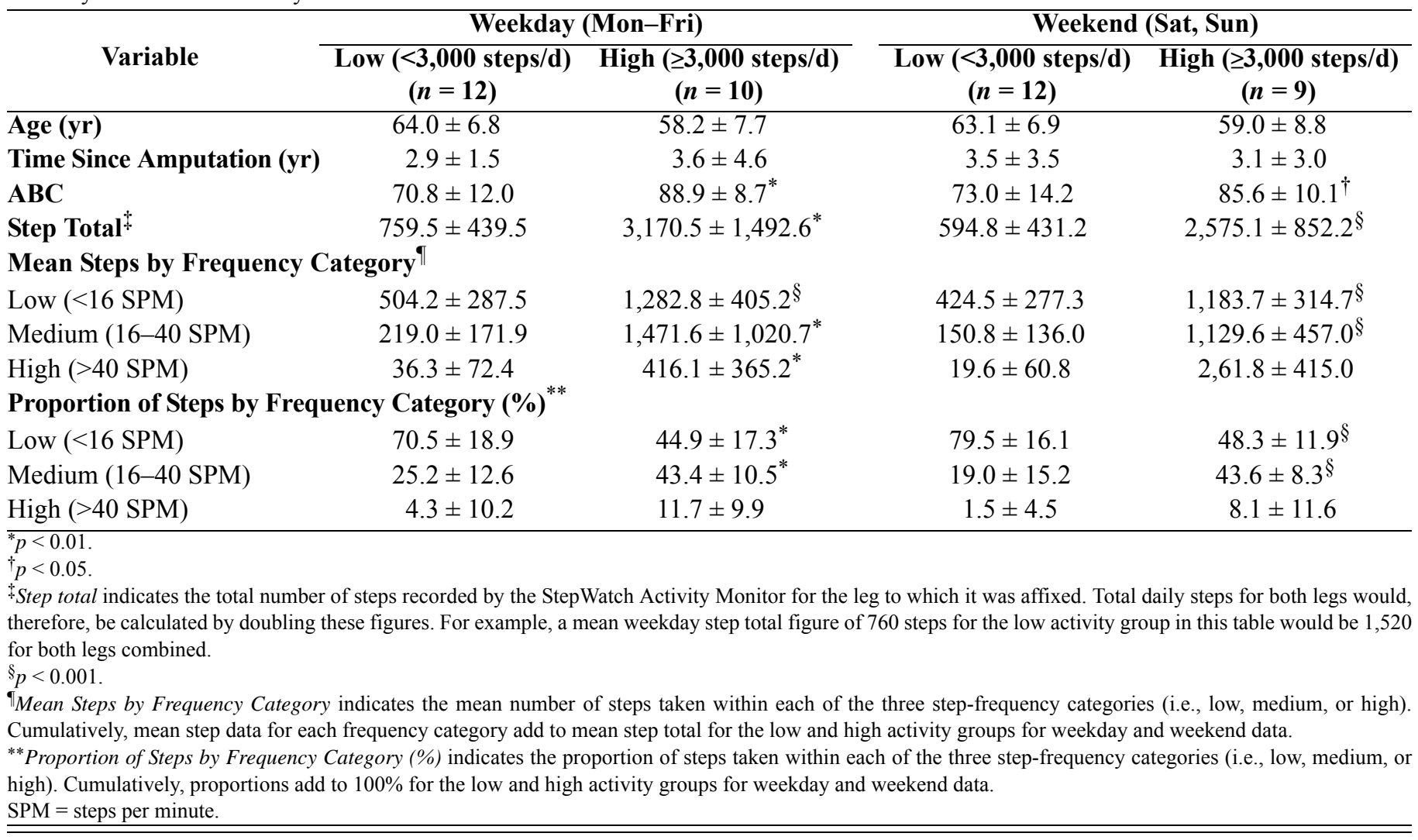

outcome for weekend activity data indicating a positive correlation between $\mathrm{ABC}$ score and step total $(r=0.46, p<$ 0.05 ) and the proportion of stepping in the high stepfrequency category $(r=0.50, p<0.05)$, as well as negative correlation between $\mathrm{ABC}$ score and the proportion of stepping in the low step-frequency category $(r=-0.44, p<$ $0.05)$.

We found a statistically significant difference for step total between the HAG and LAG for both weekday $(t(20)=$ $4.93 ;<0.01)$ and weekend data $(t(19)=6.39 ;<0.001)$. Similarly, the HAG demonstrated significantly higher weekday step counts in each of the low $(<16$ SPM), medium (16-40 SPM), and high ( $>40$ SPM) step frequency categories $(p<0.01$ for all). This result was reflected in the weekend data for low and medium step-frequency categories $(p<0.001$ for both), but not for the high step-frequency category $(p>0.05)$.

Examination of quality of stepping activity further distinguishes the HAG and LAG. The weekday data showed that 71 percent of the LAG's stepping was done at a rate of $<16$ SPM compared with only 45 percent for the HAG $(p<0.01)$. This relationship was reversed, however, for stepping in the medium step-frequency range: 44 percent of the HAG's stepping was done at a rate of 16-40 SPM compared with only 25 percent for the LAG $(p<0.01)$. We also found this relationship in the weekend stepping data: 80 percent of the LAG's stepping did not exceed a rate of 16 SPM compared with only 48 percent for the HAG $(p<0.001)$. In the medium step-frequency range, however, 44 percent the HAG's stepping was done at a rate of 16-40 SPM compared with only 19 percent for the LAG $(p<0.001)$.

\section{DISCUSSION}

The outcome of this study resulted in the rejection of the null hypothesis, thus indicating positive correlation between self-perceived balance confidence and communitybased physical activity among nonfaller patients with TTA. Consistent with previous findings, subjects in the LAG were significantly less confident in their balance ability 


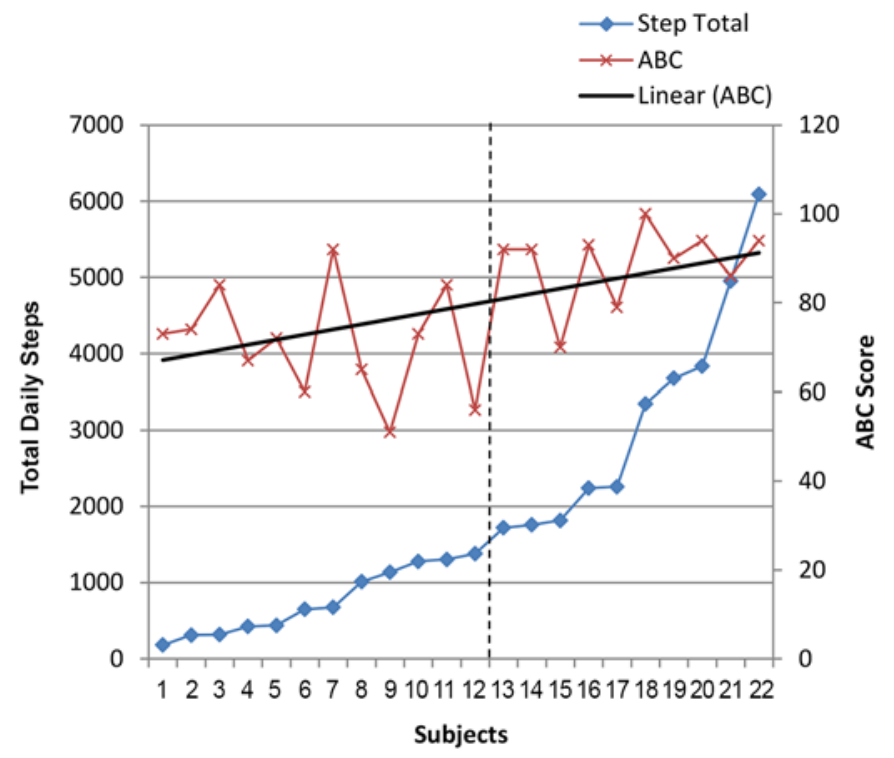

Figure.

Mean weekday total daily step count (primary $y$-axis) plotted with Activities-Specific Balance Confidence Scale (ABC) score (secondary $y$-axis) for individual subjects. A linear trend line has been included with $A B C$ scores to show the positive correlation between step count and $A B C$ score. The vertical dashed line indicates the delineation point between low $(<3,000$ steps/ d) and high ( $\geq 3,000$ steps/d) activity groups.

than those in the HAG, even though there was no difference between groups in age or time since amputation [7].

\section{Physical Activity Level}

Overall, findings from this study demonstrate a broad range in the level of physical activity performed by nonfaller individuals with TTA. Our sample population displayed physical activity levels that are both above and below 3,000 steps/d, which has been shown to be the average number of steps taken per day by an individual with a TTA with his or her prosthetic device [15]. Although the subjects for whom this average was calculated differ from our sample both in reason for amputation (primarily traumatic) and time since amputation (3$27 \mathrm{yr}$ postamputation), given the current dearth of research exploring physical activity levels among individuals with $L L$ amputation, we used this average to distinguish between the HAG and LAG. The LAG $(n=12)$ contained roughly the same number of subjects as the HAG $(n=10)$, although the HAG performed 4.2 times greater average weekday ambulatory activity. We found a slight shift from weekdays to weekends in activity-level categorization for two subjects from the LAG and two from the HAG, possibly indicating a change in vocational or recreational pursuits from a weekday schedule to a less formalized weekend schedule.

In addition to possible masking of actual fall risk, the LAG is also of concern in terms of the greater likelihood of onset of conditions typically associated with a sedentary lifestyle. Specifically, long-term inactivity may predispose an individual to develop a host of medical issues, including obesity and diabetes [23]. Previous research has indicated that increasing one's level of physical activity decreases fall risk and safeguards against injury [24]. Additionally, prolonged engagement in an inactive lifestyle may put individuals at a higher risk for falling when they do physically exert themselves because of the muscle deconditioning and weakness associated with prolonged sedentariness [7].

\section{Balance Confidence}

This study found that balance confidence differed significantly between the HAG and LAG during both the week and weekend. Further analysis indicated that balance confidence was positively related to subjects' physical activity levels. Given the sizable difference in balance confidence scores between the HAG and LAG, the results suggest that balance confidence may contribute to an individual's decision to engage in physical activity. These results are consistent with findings from previous studies linking balance confidence with mobility capacity/performance [6] and prosthetic use [25-26].

Similar to our findings, previous research has demonstrated that a subgroup of the older adult population actively avoids activities because of fear of falling [27]. Such avoidance is not without merit as a fall-reduction strategy, because individuals with low fall-related selfefficacy have been shown to be at an increased risk of falling [28]. Paradoxically, patients with LL amputation and high balance ability have been shown to be at an increased risk of falling [10], suggesting risk-taking behavior consistent with a sense of balance confidence that is discordant with actual balance ability. The question then becomes what, if any, interventions can be made available to promote engagement in physical activity by enhancing patients' fall-related self-efficacy and reducing fear of falling? Though interventions designed to improve balance outcomes in older people have demonstrated limited effectiveness [29], community-based 
exercise programs designed to improve functional capacity and reduce the risk of falls have been shown to reduce the rate of falls and risk of falling [30] and possibly fallrelated injuries [31]. There remains a lack of evidence that such interventions are effective in reducing fear of falling among older adults in general [32] and patients with LL amputation in particular.

One exception is a study of patients with transfemoral amputation (TFA) who underwent hip abductor strength training [33]. Patients with TFA who received twice weekly hip abductor strength training or arm ergometry for 8 wk demonstrated significant improvements in $\mathrm{ABC}$ scores as well as the Timed Up and Go test scores, 2 min walk test scores, and strength measures, suggesting that balance confidence can be modified and that it can improve in response to the application of a training stimulus designed to improve physical function. Where previous studies of patients with LL amputation did not find a relationship between walking ability and balance confidence [34], the success of the hip abductor training program may be attributed to the fact that it targeted a muscle group known both to contribute to the ability to ambulate and undergo detraining postamputation. Such an intervention would be particularly applicable to the LAG in the current study, which scored an average 70.8 and 73.0 percent on the $\mathrm{ABC}$ when segmented by weekday and weekend activity data, respectively, well below the 80 percent threshold on the $\mathrm{ABC}$ that indicates individuals who may benefit from an intervention to improve balance confidence [35].

\section{CONCLUSIONS}

This study adds to the available evidence regarding the relationship between balance confidence and physical activity level given its exclusive focus on nonfallers and its use of an objective measure of physical activity. It demonstrates a clear relationship between balance confidence and levels of physical activity and highlights the importance of not leaping to the conclusion that someone who has not fallen in the previous several months is a successful ambulator and not necessarily at risk of falling. In addition to standard practice, routine outpatient management of patients with LL amputation should include probing questions regarding general levels of activity to inform a clinical assessment of actual fall risk. Such probing may reveal minimal ambulatory activity levels, suggesting a strategy of limited exposure to ambu- lation-related fall risk through an intentional strategy of nonengagement in physical activity [9]. In addition, routine measurement of balance confidence should be considered. Where indicated, patients should be counseled regarding fall risk mediation strategies and the benefits of physical exercise in maintaining functional capacity and general health. Further, fall counseling should focus not on traditional fall prevention messaging, but on the benefits of maintaining health and independence because focusing on traditional fall-prevention may exacerbate fear of falling among older adults with compromised balance confidence [36].

This study has a number of limitations. A causal relationship between activity levels and falls was beyond its scope. The directionality of the relationship between balance confidence and activity levels cannot be discerned from the present study. That is, we cannot say that low balance confidence encourages patients to actively avoid physical activity or that compromised physical capacity undermines patients' confidence in their ability to engage in physical activity without an undue level of risk of falling. A prospective study with a larger sample size, ideally capturing periods both pre- and postamputation, would better elucidate whether a causal relationship exists. Further, we cannot fully assess the contribution of previous falls to either balance confidence or physical activity levels based on the study data because we inquired only of falls occurring within the past 6 mo. We selected a 6 mo window of previous fall history to facilitate accurate recall. Finally, because we measured activity during a single data collection period of 7 consecutive days, we are unable to conclude that the observed activity profiles indicate a stable pattern of activity. Rather, it could be argued that the observed activity profiles may be transient and dependent on factors unrelated to falls or balance confidence; thus, a given subject's activity level may depend on when the measures were taken relative to other life events (e.g., employment, illness). Future studies should consider whether patients with LL amputation actively limit engagement in physical activity as a fall risk-mitigation strategy and investigate the appropriate intervention to address balance confidence.

\section{ACKNOWLEDGMENTS}

\section{Author Contributions:}

Study concept and design: A. Mandel, K. Paul, M. Devlin, S. Dilkas, T. Pauley. 
Data collection: A. Mandel, K. Paul, R. Paner.

Manuscript approval: A. Mandel, K. Paul, R. Paner, M. Devlin, S. Dilkas. Academic supervisor, data analysis, manuscript preparation: T. Pauley. Financial Disclosures: The authors have declared that no competing interests exist.

Funding/Support: This material was unfunded at the time of manuscript preparation.

Institutional Review: This study was approved by the West Park Healthcare Centre, York, Ontario, Canada, and University of Toronto Research Ethics Boards, Toronto, Ontario, Canada. All study participants provided written or verbal (recorded) informed consent.

Participant Follow-Up: Study subjects were informed both in writing and verbally that the data may be published by a professional medicine journal and/or presented at scientific conferences. Subjects will not be contacted following publication of the study.

\section{REFERENCES}

1. Bussmann JB, Schrauwen HJ, Stam HJ. Daily physical activity and heart rate response in people with a unilateral traumatic transtibial amputation. Arch Phys Med Rehabil. 2008;89(3):430-34. [PMID:18295619] http://dx.doi.org/10.1016/j.apmr.2007.11.012

2. Couture M, Caron CD, Desrosiers J. Leisure activities following a lower limb amputation. Disabil Rehabil. 2010; 32(1):57-64. [PMID:19925277] http://dx.doi.org/10.3109/09638280902998797

3. Zidarov D, Swaine B, Gauthier-Gagnon C. Life habits and prosthetic profile of persons with lower-limb amputation during rehabilitation and at 3-month follow-up. Arch Phys Med Rehabil. 2009;90(11):1953-59. [PMID:19887223] http://dx.doi.org/10.1016/j.apmr.2009.06.011

4. Kulkarni J, Wright S, Toole C, Morris J, Hirons R. Falls in patients with lower limb amputations: Prevalence and contributing factors. Physiotherapy. 1996;82(2):130-36. http://dx.doi.org/10.1016/S0031-9406(05)66968-4

5. Vanicek N, Strike S, McNaughton L, Polman R. Gait patterns in transtibial amputee fallers vs. non-fallers: Biomechanical differences during level walking. Gait Posture. 2009;29(3):415-20. [PMID:19071021] http://dx.doi.org/10.1016/j.gaitpost.2008.10.062

6. Miller WC, Deathe AB, Speechley M, Koval J. The influence of falling, fear of falling, and balance confidence on prosthetic mobility and social activity among individuals with a lower extremity amputation. Arch Phys Med Rehabil. 2001;82(9):1238-44. [PMID:11552197] http://dx.doi.org/10.1053/apmr.2001.25079

7. Miller WC, Speechley M, Deathe AB. Balance confidence among people with lower-limb amputations. Phys Ther. 2002;82(9):856-65. [PMID:12201800]

8. Miller WC, Speechley M, Deathe B. The prevalence and risk factors of falling and fear of falling among lower extremity amputees. Arch Phys Med Rehabil. 2001;82(8):
1031-37. [PMID:11494181]

http://dx.doi.org/10.1053/apmr.2001.24295

9. Tinetti ME, Richman D, Powell L. Falls efficacy as a measure of fear of falling. J Gerontol. 1990;45(6):P239-43. [PMID:2229948] http://dx.doi.org/10.1093/geronj/45.6.P239

10. Wong CK, Chen CC, Blackwell WM, Rahal RT, Benoy SA. Balance ability measured with the Berg Balance Scale: A determinant of fall history in community-dwelling adults with leg amputation. J Rehabil Med. 2015;47(1):80-86. [PMID:25223891]

11. Dite W, Connor HJ, Curtis HC. Clinical identification of multiple fall risk early after unilateral transtibial amputation. Arch Phys Med Rehabil. 2007;88(1):109-14. [PMID:17207685] http://dx.doi.org/10.1016/j.apmr.2006.10.015

12. O’Loughlin JL, Robitaille Y, Boivin JF, Suissa S. Incidence of and risk factors for falls and injurious falls among the community-dwelling elderly. Am J Epidemiol. 1993;137(3): 342-54. [PMID:8452142]

13. Marques AP, Mendes YC, Taddei U, Pereira CA, Assumpção A. Brazilian-Portuguese translation and cross cultural adaptation of the activities-specific balance confidence (ABC) scale. Braz J Phys Ther. 2013;17(2):170-78. [PMID:23778771] http://dx.doi.org/10.1590/S1413-35552012005000072

14. Coleman KL, Smith DG, Boone DA, Joseph AW, del Aguila MA. Step activity monitor: Long-term, continuous recording of ambulatory function. J Rehabil Res Dev. 1999;36(1): 8-18. [PMID:10659890]

15. Klute GK, Berge JS, Orendurff MS, Williams RM, Czerniecki JM. Prosthetic intervention effects on activity of lower-extremity amputees. Arch Phys Med Rehabil. 2006;87(5):717-22. [PMID:16635636] http://dx.doi.org/10.1016/j.apmr.2006.02.007

16. Stepien JM, Cavenett S, Taylor L, Crotty M. Activity levels among lower-limb amputees: Self-report versus step activity monitor. Arch Phys Med Rehabil. 2007;88(7):896-900. [PMID:17601471] http://dx.doi.org/10.1016/j.apmr.2007.03.016

17. Mudge S, Taylor D, Chang O, Wong R. Test-retest reliability of the StepWatch Activity Monitor outputs in healthy adults. J Phys Act Health. 2010;7(5):671-76. [PMID:20864764]

18. Mudge S, Stott NS. Test-retest reliability of the StepWatch Activity Monitor outputs in individuals with chronic stroke. Clin Rehabil. 2008;22(10-11):871-77. [PMID:18955419] http://dx.doi.org/10.1177/0269215508092822

19. Parker K, Kirby RL, Adderson J, Thompson K. Ambulation of people with lower-limb amputations: Relationship between capacity and performance measures. Arch Phys Med Rehabil. 2010;91(4):543-49. [PMID:20382285] http://dx.doi.org/10.1016/j.apmr.2009.12.009 
20. Powell LE, Myers AM. The Activities-specific Balance Confidence (ABC) Scale. J Gerontol A Biol Sci Med Sci. 1995;50A(1):M28-34. [PMID:7814786] http://dx.doi.org/10.1093/gerona/50A.1.M28

21. Miller WC, Deathe AB, Speechley M. Psychometric properties of the Activities-specific Balance Confidence Scale among individuals with a lower-limb amputation. Arch Phys Med Rehabil. 2003;84(5):656-61. [PMID:12736877] http://dx.doi.org/10.1016/S0003-9993(02)04807-4

22. Sakakibara BM, Miller WC, Backman CL. Rasch analyses of the Activities-specific Balance Confidence Scale with individuals 50 years and older with lower-limb amputations. Arch Phys Med Rehabil. 2011;92(8):1257-63.

[PMID:21704978]

http://dx.doi.org/10.1016/j.apmr.2011.03.013

23. Sullivan PW, Morrato EH, Ghushchyan V, Wyatt HR, Hill JO. Obesity, inactivity, and the prevalence of diabetes and diabetes-related cardiovascular comorbidities in the U.S., 2000-2002. Diabetes Care. 2005;28(7):1599-1603. [PMID:15983307] http://dx.doi.org/10.2337/diacare.28.7.1599

24. Pereira CL, Baptista F, Infante P. Role of physical activity in the occurrence of falls and fall-related injuries in community-dwelling adults over 50 years old. Disabil Rehabil. 2014;36(2):117-24. [PMID:23594055] http://dx.doi.org/10.3109/09638288.2013.782355

25. Wong CK, Young RS, Ow-Wing C, Karimi P. Determining $1-y$ prosthetic use for mobility prognoses for communitydwelling adults with lower-limb amputation: Development of a clinical prediction rule. Am J Phys Med Rehabil. 2016. [PMID:26390393] http://dx.doi.org/10.1097/PHM.0000000000000383

26. Wong CK, Chen CC, Benoy SA, Rahal RT, Blackwell WM. Role of balance ability and confidence in prosthetic use for mobility of people with lower-limb loss. J Rehabil Res Dev. 2014;51(9):1353-64. [PMID:25785790] http://dx.doi.org/10.1682/JRRD.2013.11.0235

27. Tinetti ME, Mendes de Leon CF, Doucette JT, Baker DI. Fear of falling and fall-related efficacy in relationship to functioning among community-living elders. J Gerontol. 1994;49(3):M140-47. [PMID:8169336] http://dx.doi.org/10.1093/geronj/49.3.M140

28. Cumming RG, Salkeld G, Thomas M, Szonyi G. Prospective study of the impact of fear of falling on activities of daily living, SF-36 scores, and nursing home admission. J Gerontol A Biol Sci Med Sci. 2000;55(5):M299-305. [PMID:10819321] http://dx.doi.org/10.1093/gerona/55.5.M299

29. Howe TE, Rochester L, Neil F, Skelton DA, Ballinger C. Exercise for improving balance in older people. Cochrane Database Syst Rev. 2011;(11):CD004963. [PMID:22071817]

30. Gillespie LD, Robertson MC, Gillespie WJ, Sherrington C, Gates S, Clemson LM, Lamb SE. Interventions for prevent- ing falls in older people living in the community. Cochrane Database Syst Rev. 2012;9:CD007146. [PMID:22972103] http://dx.doi.org/10.1002/14651858.CD007146.pub3

31. Health Quality Ontario. Prevention of falls and fall-related injuries in community-dwelling seniors: An evidencebased analysis. Ont Health Technol Assess Ser. 2008;8(2): 1-78. [PMID:23074507]

32. Kendrick D, Kumar A, Carpenter H, Zijlstra GA, Skelton DA, Cook JR, Stevens Z, Belcher CM, Haworth D, Gawler SJ, Gage H, Masud T, Bowling A, Pearl M, Morris RW, Iliffe S, Delbaere K. Exercise for reducing fear of falling in older people living in the community. Cochrane Database Syst Rev. 2014;11:CD009848. [PMID:25432016] http://dx.doi.org/10.1002/14651858.CD009848.pub2

33. Pauley T, Devlin M, Madan-Sharma P. A single-blind, crossover trial of hip abductor strength training to improve Timed Up \& Go performance in patients with unilateral, transfemoral amputation. J Rehabil Med. 2014;46(3):264-70.

[PMID:24363039] http://dx.doi.org/10.2340/16501977-1270

34. Miller WC, Deathe AB. The influence of balance confidence on social activity after discharge from prosthetic rehabilitation for first lower limb amputation. Prosthet Orthot Int. 2011;35(4):379-85. [PMID:21846808] http://dx.doi.org/10.1177/0309364611418874

35. Myers AM, Fletcher PC, Myers AH, Sherk W. Discriminative and evaluative properties of the Activities-Specific Balance Confidence (ABC) scale. J Gerontol A Biol Sci Med Sci. 1998;53(4):M287-94. [PMID:18314568] http://dx.doi.org/10.1093/gerona/53A.4.M287

36. Hughes K, van Beurden E, Eakin EG, Barnett LM, Patterson E, Backhouse J, Jones S, Hauser D, Beard JR, Newman B. Older persons' perception of risk of falling: Implications for fall-prevention campaigns. Am J Public Health. 2008; 98(2):351-57. [PMID:18172132] http://dx.doi.org/10.2105/AJPH.2007.115055

Submitted for publication March 12, 2015. Accepted in revised form October 21, 2015.

This article and any supplementary material should be cited as follows:

Mandel A, Paul K, Paner R, Devlin M, Dilkas S, Pauley T. Balance confidence and activity of community-dwelling patients with transtibial amputation. J Rehabil Res Dev. 2016;53(5):551-60.

http://dx.doi.org/10.1682/JRRD.2015.03.0044

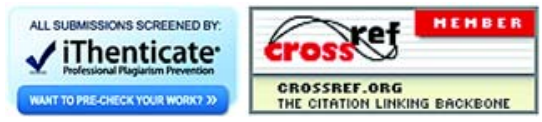


